\title{
Efficient Power Allocation for NOMA-enabled IoT Networks in 6G Era
}

\author{
Wali Ullah Khan, Furqan Jameel, Muhammad Ali Jamshed, Haris Pervaiz, \\ Shafiullah Khan and Ju Liu
}

\begin{abstract}
Due to unrivaled effectiveness, non-orthogonal multiple access (NOMA) has risen as a promising multiple access scheme for the Internet of things (IoT). In this paper, we provide a new power allocation technique for improving the energy and spectral efficiency of NOMA-enabled IoT devices. The power allocation is performed without compromising the quality of service (QoS) requirements of the network. By considering the transmit power, QoS and successive interference cancellation (SIC) constraints, we use the sequential quadratic programming (SQP) technique to solve the non-convex problem. To assess the performance of our scheme, we compare the proposed SQP-based approach with the conventional KKT-based optimization method. We provide Monte Carlo simulation results to assess our proposed power allocation framework and illustrate the performance improvements against orthogonal multiple access (OMA) scheme. The results uncover that the proposed SQP-based power optimization design substantially improves the performance of the NOMA-enabled IoT network.
\end{abstract}

\section{Index Terms}

Wali Ullah Khan and Ju Liu are with School of Information Science and Engineering, Shandong University, Qingdao 266237, People’s Republic of China. (emails: waliullahkhan30@gmail.com, juliu@sdu.edu.cn)

Furqan Jameel is with the Department of Communications and Networking, Aalto University, 02150 Espoo, Finland. (email: furqanjameel01@gmail.com)

Muhammad Ali Jamshed is with the Institute of Communication Systems (ICS), Home of 5G Innovation Centre (5GIC), University of Surrey, Stag Hill, Guildford GU2 7XH, UK. (email: m.jamshed@surrey.ac.uk)

Haris Pervaiz is with the School of Computing and Communications, Lancaster University, Bailrigg, Lancaster LA1 4YW, UK. (email: h.b.pervaiz@lancaster.ac.uk)

Shafiullah Khan is with the Institute of Computing (IoC), Kohat University of Science and Technology (KUST), Kohat City, 26000, Pakistan. (email: skkust@gmail.com) 
Internet of Things, Non-orthogonal multiple access, 6G, Radio resource management and optimization methods.

\section{INTRODUCTION}

The number of devices connected to the Internet is increasing every year. According to one estimate, the number of connected devices will reach up to 30 billion by 2025 , increasing the bandwidth, reliability and latency requirements [1]. Eventually, these devices will be commonly known as the Internet of things (IoT) that would become a key component of future smart cities. Although these devices will raise the issues of co-channel interference, link failures, and high end-to-end delays, the most challenging task would be the limited battery life of these devices [2]. The environmental concerns along with the high cost of battery management/ replacement would require advance power allocation techniques to incorporate in a city-wide communication network. These techniques would enable low-powered IoT devices to work efficiently besides enabling efficient communication in a smart city [3].

Multiple access schemes have always played a critical role in the development of largescale wireless networks. The orthogonal multiple access (OMA) techniques such as frequency division/ time division/ code division multiple access techniques (FDMA/TDMA/CDMA) have been widely deployed [4], where limited resources are reserved for each user [1]. Although they have been used to remove the impact of interference to some extent, their utility in massive and large-scale IoT networks is very limited. It is because the OMA techniques assign orthogonal resources to the devices, which though reduce inter-device interference, but does not scale up with the increasing number of devices. Thus, to enable sustainable communication in future smart cities, there is a requirement of scalable and spectral efficient access techniques that can help in meeting the unprecedented demands of the explosive growth of end users [2].

In recent years, non-orthogonal multiple access (NOMA) has emerged as a new channel access architecture to provide a spectrally efficient solution for beyond fifth-generation (5G) networks [5]. This improvement in spectral efficiency (SE) does not come at the cost of reduced security or user fairness. Several studies have proven that conventional OMA techniques under-perform when compared with the NOMA techniques, especially in terms of user fairness [6], SE [7] and secrecy capacity [8]. One of the reasons behind such improvements is the successive interference cancellation (SIC), which is performed at the receiver side [9]. Moreover, the decoding order of 
the users can be exploited to improve the fairness and throughput among users [10].

At present, the cellular traffic uses OMA techniques for devices to provide access to the Internet. As per one of the studies, NOMA techniques can be helpful in provisioning of services like massive machine-type communications (mMTC) and ultra-reliable low latency communications (URLLC) [11]. The mobile traffic would share a dominant portion of the spectrum, especially after the proliferation of IoT devices. Thus, utilization of NOMA techniques becomes all the more important for efficient bandwidth utilization and resource allocation [12]. Typically, the NOMA techniques employ the concept of non-orthogonality through the power domain variations. The power domain can perform by multiplexing the transmit data in frequency, time, and code domains [13]. This multiplexed data at the transmitter side is forwarded over the wireless channel by allocating a large power difference in the different users [14]. Subsequently, a multi-user detection scheme can be used at the receiver end, for example making use of SIC [15]. In this way, the same spectrum is used which is made possible through the superpositioncoding at the transmitter end while SIC at the receiver side. In simple words, this combination works as the receiver tries to decode the strongest user first and treats another signal as noise and interference. Afterward, it removes the most powerful signal. This removal of unintended/ interference message continues until the intended signals are obtained by all the users [2].

\section{A. Related Work}

One can argue that the novelty of NOMA lies in intelligently assigning the transmit power to a different user. This intelligent assignment is not only necessary for reliable transmission of the message but is also critical from the energy management aspect of the devices. It becomes an even more critical aspect for the low-powered miniature IoT devices with small energy sources. Due to this reason, energy efficiency (EE) techniques have been investigated in conventional NOMA systems. A multi-user downlink NOMA technique was proposed by the authors of [16]. Here, the authors discussed different applications of coordinated multi-point NOMA techniques for downlink transmission of signals. The authors of [17] used Alamouti codes for downlink NOMA transmission in cluster-based cells. Their target was to provide a reasonable data rate for edge user without compromising the near user. In [18], the authors proposed a grouping mechanism for low-powered edge users. Resultantly, they provide a closed-form solution of outage probability for the opportunistic NOMA system with power optimization in multi-cell 
users. Similarly, the authors of [19] proposed a suboptimal scheduling strategy while the authors of [20] utilized the relaying signal to allocate power among different cells. In [21], the authors addressed the problem of dynamic power control to enhance the users' sum capacity and reduce the total transmission power. They considered a homogenous communication environment where the NOMA users form the cluster of two users. The resource management problems for multi-cell multiple-input-multiple-output (MIMO) networks were investigated by the authors of [22]-[25]. In particular, the authors in [22], [23] provided a suboptimal scheme to maximize the users' sum capacity. Their results showed that even using the suboptimal approaches can result in significant improvements in user capacity of NOMA systems. From the perspective of EE optimization, the authors of [24], [25] investigated EE, outage probability and effective capacity of the system. Similarly, Shi et al. presented a suboptimal technique to handle the non-convex optimization problem [26]. In addition to this, resource management problems for EE maximization were also investigated in NOMA-based heterogeneous networks by the authors of [27], [28]. In particular, the authors of [27] investigated an efficient power allocation in two-tier heterogeneous network to maximize the EE of small-cell. The problem was first transformed into convex optimization using sequential convex programing and then employed closed-form expression to obtain an efficient solution. In [28], a problem of sub-channel assignment and power allocation was explored to maximize the EE of macro-cell and small-cells. A suboptimal techniques of convex relaxation and dual decomposition method were proposed to solve the non-convex optimization problem.

Moreover, EE schemes were also investigated in NOMA-enabled IoT networks. For example, Zhai et al. [29] investigated an optimization problem of dynamic user scheduling and power control in IoT networks. The problem was formulated as a stochastic optimization and the objective was to reduce to power consumption of the network. The authors presented a low complexity algorithm based on branch and bound to find the efficient power allocation. In [30], a problem of efficient resource management was considered to maximize the EE of the NOMAenabled IoT network with energy harvesting. They proposed an efficient algorithm based on mesh adaptive direct search to obtain the optimal solution. In [31], Sikeridis et al. provide energy efficient communication framework in wireless powered IoT network supporting by power domain NOMA. The authors employed reinforcement learning for sensing operation and explored an efficient power allocation method based on Nash equilibrium to improve the overall system performance. In [32], the problem of resource allocation for EE with nonlinear energy harvesting 
was also investigated in machine-to-machine based communication. The purpose was to reduce the power consumption of the entire network through joint optimization of transmit power and time resources. To get the efficient solution, the authors first transformed the problem and then applied an iterative algorithm for efficient power control and time allocation. Reference [33] adopted two-step approach for EE in NOMA-enabled mobile edge computing. For a given task edge-server, the authors first provided an efficient solution for joint formulation of rate allocation task offloading and time allocation. Then, based on the above solution, an efficient task edgeserver assignment technique was proposed to further reduce the overall power consumption. Han et al. in [34] optimized the joint computation and resource management to increase the secrecy EE of computation offloading of mMTC. They adopted matching theory for efficient sub-channel allocation and derived closed-form expression for suboptimal power control. Finally, the authors of [35] proposed the EE resource management scheme in hybrid time-division-multiple-accessNOMA network. The optimization problem was first transformed using nonlinear fractional programing method and then Dinkelbach's and Lagrangian duality methods were adopted to obtain the efficient solutions.

\section{B. Motivation and Contributions}

Motivated by the aforementioned advances, this paper sheds light on the power optimization framework for NOMA-enabled IoT devices. The aim is to minimize the total transmitted power of IoT devices under the SIC constraint. For a particular sub-channel assignment, we employ a new approach based on sequential quadratic programming (SQP) to solve the non-convex power allocation problem. Through extensive simulations, the effectiveness of the proposed approach is highlighted pinpointing that the proposed SQP-based approach significantly outperforms the suboptimal NOMA scheme for IoT devices. The main contributions of this work are summarized as follows:

- A NOMA-enabled IoT network is considered, where a single base station (BS) serves $K$ IoT devices through $M$ number of sub-channels. A novel optimal power allocation approach is formulated to maximize the total EE of IoT devices. The problem is formulated subject to the constraints such as the maximum number of IoT devices on each sub-channel, individual QoS requirements, maximum transmit power and the minimum gap among different IoT devices on each sub-channel to guarantee the SIC operation. 
Table I

LIST OF NOTATIONS

\begin{tabular}{|c|c|}
\hline Symbols & Definition \\
\hline$K$ & The set of IoT devices \\
\hline$M$ & The set of sub-channels \\
\hline $\mathcal{S}_{m}$ & The set of IoT devices communicating on sub-channel $m$ \\
\hline$y_{k, m}$ & The received signal of IoT device $k$ on sub-channel $m$ \\
\hline$p_{l, m}$ & The transmit power of IoT device $l$ on sub-channel $m$ \\
\hline$h_{k, m}$ & The channel coefficient of IoT device $k$ on sub-channel $m$ \\
\hline$\rho^{2}$ & Variance of additive white Gaussian noise \\
\hline$R_{k, m}$ & The data rate of IoT device $k$ on sub-channel $m$ \\
\hline$x_{l, m}$ & The data symbol of IoT device $l$ on sub-channel $m$ \\
\hline$\xi_{k, m}$ & The signal to interference plus noise ratio of IoT device $k$ on sub-channel $m$ \\
\hline$\beta$ & The minimum gap between IoT devices powers in $\mathcal{S}_{m}$ to perform the successful SIC technique \\
\hline $\mathcal{S}_{\max }$ & Maximum number of IoT devices on sub-channel $m$ at one time \\
\hline$\Gamma_{\min }$ & The minimum threshold to guarantee the IoT's QoS requirements \\
\hline $\mathcal{P}_{\max }$ & The total power budget of BS \\
\hline$\Pi$ & Represents the Jacobian matrix \\
\hline$\nu_{k, m}$ & The additive white Gaussian noise of IoT device $k$ on sub-channel $m$ \\
\hline$\amalg$ & Represents the Hessian matrix \\
\hline $\mathcal{L}()$. & Represents the Lagrange function \\
\hline$\lambda_{k}, \eta_{k}, \pi_{k}$ & Represents the Lagrange multipliers \\
\hline$\Upsilon$ & A matrix consists of a Jacobian matrix, a Hessian matrix, and a Zero matrix \\
\hline$\psi$ & The non-negative step size \\
\hline$\alpha$ & Represents the correction vector \\
\hline$\delta \mathcal{L}()$. & Represents the Gradient of Lagrange function \\
\hline$z$ & Represents the iteration index \\
\hline
\end{tabular}

- In contrast to the previously discussed studies from the current state of the art, which does not guarantee the successful SIC process at the receiver side, a constraint to ensure the minimum gap among different IoT devices for successful SIC is considered. Moreover, most of the existing related research works proposed suboptimal approaches to solve their considered framework. Adopting different approach from the literature, a novel non-convex optimization technique known as SQP is exploited to obtain a reliable and efficient solution. In order to evaluate the performance of the proposed approach, a benchmark Karush-Kuhn-Tucker 


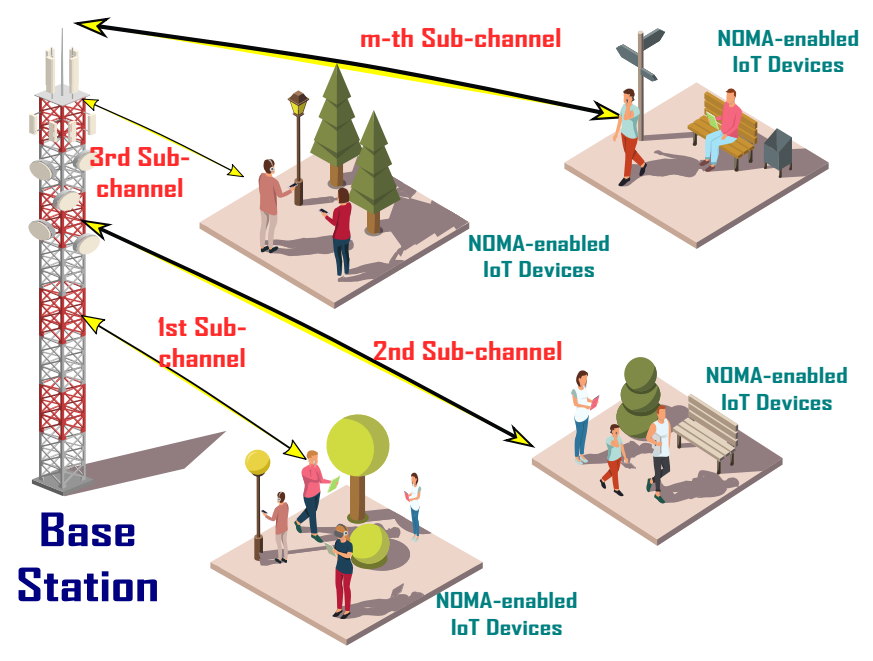

Figure 1. NOMA-enabled IoT network.

(KKT)-based suboptimal approach is also analyzed where the Lagrangian multipliers are updated using the sub-gradient method. The performance of the proposed approach is evaluated with the aid of Monte Carlo based simulations.

- It can be observed that the proposed SQP-based optimal power allocation approach significantly improves the performance of the NOMA-enabled IoT network, in contrast to the benchmark KKT-based suboptimal power allocation approach. More specifically, by varying the different system parameters of the network such as increasing number of IoT devices, increasing values of transmit power and circuit power $\left(P_{C}\right)$ consumption at $\mathrm{BS}$, the proposed optimal SQP-based approach significantly outperforms the suboptimal benchmark KKT-based approach and the conventional OMA in terms of system spectral and energy efficiency.

\section{Paper Organization}

The remainder of this article is organized as follows. In Section II, details of the network model along with problem formulation steps have been given. Section III discusses the SQPbased proposed solution. Section IV presents results and discussions while Section V provides concluding remarks. Table I represents the different symbols used in the paper. 


\section{Network Model ANd Problem Formulation}

As shown in Fig. 1, an IoT wireless network is considered, where a base station (BS) serves $K$ IoT devices through $M$ sub-channels. An outdoor communication scenario is assumed with a serving BS and multiple NOMA-enabled IoT devices. Generally, this type of communication can take place between different smart watches, or other wearable devices connected to an RF transmission source. The downlink NOMA transmission scheme is considered and it is assumed that the information of all IoT devices is available at the BS. In addition, the transmitter and the IoT devices are assumed to be equipped with omni-directional antenna. All the wireless links follow independent and identically distributed (i.i.d) Rayleigh fading. It is also assumed that each sub-channel can accommodate multiple IoT devices at one time [36]. If $\mathcal{S}_{m}$ denotes the set of IoT devices on sub-channel $m \in M$ and $x_{l, m}$ is the signal of IoT device $l \in \mathcal{S}_{m}$, the received signal of IoT device $k \in \mathcal{S}_{m}$ on sub-channel $m$ is

$$
y_{k, m}=h_{k, m} \sum_{l=1}^{\mathcal{S}_{m}} \sqrt{p_{l, m}} x_{l, m}+\nu_{k, m},
$$

where $h_{k, m}$ in (1) is the channel coefficient of IoT device $k$ on sub-channel $m, p_{l, m}$ denotes the transmit power of IoT device $l$ on sub-channel $m, x_{l, m}$ is the data signal of IoT device $l$ on sub-channel $m$ and $\nu_{k, m}$ shows the additive white Gaussian noise of IoT device $k$ on subchannel $m$ with zero mean and variance $\rho^{2}$. As already mentioned that a sub-channel $m$ can accommodate $\mathcal{S}_{m}$ IoT devices at one time, an IoT device $k$ can apply SIC technique for IoT device $l$ if $\left|h_{l, m}\right|^{2} \leq\left|h_{k, m}\right|^{2}$. However, an IoT device $k$ cannot apply SIC for IoT device $l$ if $\left|h_{l, m}\right|^{2} \geq\left|h_{k, m}\right|^{2}$. By considering the decoding complexity of SIC at the receiver side, it is assumed that at a sub-channel $m$ can accommodate at most $\mathcal{S}_{\max }$ IoT devices at one time, i.e., $\left|\mathcal{S}_{m}\right| \leq \mathcal{S}_{\max }$. Based on these observations, the rate of IoT device $k$ on sub-channel $m$ can be expressed as

$$
R_{k, m}=\log _{2}\left(1+\xi_{k, m}\right),
$$

where $\xi_{k, m}$ in (2) is the signal to interference plus noise ratio defined as follow:

$$
\xi_{k, m}=\frac{p_{k, m}\left|h_{k, m}\right|^{2}}{\left|h_{k, m}\right|^{2} \sum_{l=1}^{k-1} p_{l, m}+\rho^{2}} .
$$

NOMA in power domain multiplexing requires successful SIC process at the receivers [6]. To do so, we introduce a SIC constraint in our proposed framework such that the minimum gap 
between IoT devices' power in $\mathcal{S}_{m}$ on sub-channel $m$ should satisfy the following condition as follow:

$$
p_{k, m}\left|h_{k-1, m}\right|^{2}-\sum_{l=1}^{k-1} p_{l, m}\left|h_{k-1, m}\right|^{2} \geq \beta .
$$

where $\beta$ in (4) depicts the minimum gap between IoT devices' power in $\mathcal{S}_{m}$ to perform the successful SIC technique.

In this work, the system performance is evaluated in terms of total achievable EE subject to each IoT device QoS, transmit power and successful SIC constraints. Additionally, we introduce a binary variable $\varpi_{k, m}$ which indicates whether a sub-channel $m$ is assigned to IoT device $k$. Thus, our objective is to minimize the transmit power of all IoT devices at the source through optimization of $\left\{p_{k, m}, \varpi_{k, m}\right\}$. This can be achieved by investigating the following optimization problem as

$$
\begin{array}{ll}
\text { (P) } & \operatorname{minimize}_{\varpi_{k, m}, p_{k, m}} \sum_{m=1}^{M} \sum_{k=1}^{K} \varpi_{k, m} p_{k, m} \\
\text { s.t. } & \sum_{k=1}^{K} \varpi_{k, m} \leq \mathcal{S}_{\max }, \forall m, \\
& \varpi_{k, m} R_{k, m} \geq \Gamma_{\min }, \forall m, \forall k, \\
& \sum_{m=1}^{M} \sum_{k=1}^{K} \varpi_{k, m} p_{k, m} \leq P_{\max }, \\
& \sum_{m=1}^{M} \varpi_{k, m}\left(\sum_{l=1}^{k-1} p_{l, m}+\frac{\beta}{\left|h_{k-1, m}\right|^{2}} \leq p_{k, m}\right), \forall k, \\
& \varpi_{k, m} p_{k, m} \geq 0, \forall m, \forall k, \\
& \varpi_{k, m} \in\{0,1\}, \forall m, \forall k .
\end{array}
$$

where constraint (5a) limit the number of IoT devices on sub-channel $m$ at one time. Constraint (5b) satisfies the QoS of IoT device $k$ on sub-channel $m$, where $\Gamma_{\min }$ shows the minimum threshold for user's QoS requirements. Constraint $(5 \mathrm{c})$ ensures that the sum power of all IoT devices should be less than or equal to the total power of the $\mathrm{BS}$, where $P_{\max }$ represents the total power budget at BS. In addition, constraint in (5d) makes the SIC process successful. The last constraint in (5e) shows the non-negative powers of IoT devices. 


\section{PROpOSED SOLUTIONS}

As expressed in $(\mathrm{P})$, the optimal resource allocation problem for the considered communication scenario is mixed integer non-convex because of the binary variable and interference term in the rate expression [37]. Thus, it hard to obtain the joint solution. In this work, it is assumed that the sub-channels to IoT devices are allocated prior in an independent manner before the power allocation mechanism ${ }^{1}$. Therefore, the original problem $(\mathrm{P})$ can be simplified to power allocation problem as follow:

$$
\begin{array}{ll}
\text { (P1) } & \underset{p_{k, m}}{\operatorname{minimize}} \sum_{m=1}^{M} \sum_{k=1}^{K} p_{k, m} \\
\text { s.t. } & R_{k, m} \geq \Gamma_{\min }, \forall m, \forall k, \\
& \sum_{m=1}^{M} \sum_{k=1}^{K} p_{k, m} \leq P_{\max }, \\
& \sum_{l=1}^{k-1} p_{l, m}+\frac{\beta}{\left|h_{k-1, m}\right|^{2}} \leq p_{k, m}, \forall m, \forall k, \\
& p_{k, m} \geq 0, \forall m, \forall k .
\end{array}
$$

The optimization problem (P1) is still non-convex problem. Therefore, we employ a new SQP-based approach which has been extensively used as an efficient method to solve the nonconvex optimization problems [39]-[41]. Additionally, a benchmark KKT-based low complexity suboptimal solution is also provided to compare the effectiveness of the proposed SQP-based approach.

\section{A. SQP-based Optimal Power Allocation}

Here, we provide SQP-based approach for energy efficient power allocation to solve the optimization problem (P1) formulated in Eq. (6). The SQP is an optimization approach for modeling nonlinear programming problem into quadratic programming sub-problems. To exploit SQP-based approach, we first derive a Jacobian matrix $\Pi$, where the $a$-th and $b$-th entries on

\footnotetext{
${ }^{1}$ In this work, our major focus is towards optimizing the power allocation aspect of the NOMA-enabled IoT devices. Therefore, we consider that the sub-channels assignment to different IoT devices has already been completed [38]. The problem of efficient sub-channel allocation is set aside for our future work.
} 
sub-channel $m$ are

$$
\boldsymbol{\Pi}=\left[\begin{array}{c}
\left.\left[\frac{\partial \boldsymbol{\Gamma}_{\min }(\boldsymbol{p})}{\partial \boldsymbol{p}}\right]_{\mathcal{S}_{m} \times \mathcal{S}_{m}}^{T}\left[\frac{\partial \boldsymbol{P}_{\max }(\boldsymbol{p})}{\partial \boldsymbol{p}}\right]_{\mathcal{S}_{m} \times \mathcal{S}_{m}}^{T}\right]^{T} \\
{\left[\left[\frac{\partial \boldsymbol{\beta}_{\min , 1}(\boldsymbol{p})}{\partial \boldsymbol{p}}\right]_{\mathcal{S}_{m} \times 1}^{T}\left[\frac{\partial \boldsymbol{\beta}_{\min , o}(\boldsymbol{p})}{\partial \boldsymbol{p}}\right]_{\mathcal{S}_{m} \times\left(\mathcal{S}_{m}-1\right)}^{T}\right]_{\mathcal{S}_{m} \times \mathcal{S}_{m}}^{T}}
\end{array}\right]^{T},
$$

where

$$
\begin{gathered}
\boldsymbol{\Gamma}_{\min }(\boldsymbol{p})=\left[\begin{array}{c}
\Gamma_{\min }-\log _{2}\left(1+\frac{p_{1, m}\left|h_{1, m}\right|^{2}}{\rho^{2}}\right) \\
\Gamma_{\min }-\log _{2}\left(1+\frac{\left|h_{2, m}\right|^{2} p_{2, m}}{\left|h_{2, m}\right|^{2} p_{1, m}+\rho^{2}}\right) \\
\vdots \\
\Gamma_{\min }-\log _{2}\left(1+\frac{\left|h_{k, m}\right|^{2} p_{k, m}}{\left|h_{k, m}\right|^{2} \sum_{l=1}^{k-1} p_{l, m}+\rho^{2}}\right)
\end{array}\right], \\
\boldsymbol{\beta}_{\min }(\boldsymbol{p})=\left[\begin{array}{c}
p_{1, m}+\frac{\beta}{\left|h_{1, m}\right|^{2}}-p_{2, m} \\
\vdots \\
k-1 \\
\sum_{l=1} p_{l, m}+\frac{\beta}{\left|h_{k-1, m}\right|^{2}}-p_{k, m}
\end{array}\right], \\
\boldsymbol{P}_{\max }(\boldsymbol{p})=\left[\begin{array}{c}
p_{1, m}-P_{\max } \\
p_{2, m}-P_{\max } \\
\vdots \\
p_{k, m}-P_{\max }
\end{array}\right]
\end{gathered}
$$

The partial derivatives of (8), (9) and (10) can be respectively calculated as

$$
\begin{gathered}
\frac{\partial \boldsymbol{\Gamma}_{\min }(\boldsymbol{p})}{\partial p_{k, m}}=\left\{\begin{array}{cc}
\phi_{1}, & \text { if } a=b, \\
\phi_{2}, & \text { if } a>b, \\
0, & \text { if } a<b,
\end{array}\right. \\
\frac{\partial \boldsymbol{P}_{\max }(\boldsymbol{p})}{\partial p_{k, m}}=\left\{\begin{array}{cc}
0, & \text { if } a>b, \\
1, & \text { if } a=b, \\
0, & \text { if } a<b,
\end{array},\right. \\
\frac{\partial \boldsymbol{\beta}_{\min , 1}(\boldsymbol{p})}{\partial p_{k, m}}=\mathbf{0}^{T}, \quad \forall \mathcal{S}_{m},
\end{gathered}
$$


where $\mathbf{0}=[0,0,0 \ldots .$.$] , and when \forall 0>1$, then

$$
\frac{\partial \boldsymbol{\beta}_{\min , o}(\boldsymbol{p})}{\partial p_{k, m}}=\left\{\begin{array}{rl}
1, & \text { if } \quad a>b, \\
-1, & \text { if } a=b, \\
0, & \text { if } a<b,
\end{array} .\right.
$$

where $a$ and $b$ denote the row and column of the matrix. The values of $\phi_{1}$ and $\phi_{2}$ in (11) can be written as

$$
\begin{gathered}
\phi_{1}=\frac{-\left|h_{k, m}\right|^{2}}{\left|h_{k, m}\right|^{2} \sum_{l=1}^{k-1} p_{l, m}+\rho^{2}+p_{k, m}\left|h_{k, m}\right|^{2}}, \\
\phi_{2}=\frac{p_{j, m}\left|h_{j, m}\right|^{4}}{\gamma_{j, m}\left(\left|h_{j, m}\right|^{2} \sum_{l=1}^{j-1} p_{l, m}+\rho^{2}+p_{j, m}\left|h_{j, m}\right|^{2}\right)}
\end{gathered}
$$

where

$$
\gamma_{j, m}=\left|h_{j, m}\right|^{2} \sum_{l=1}^{j-1} p_{l, m}+\rho^{2} .
$$

Next, we derive a Hessian matrix which is represented by $\mathbf{\amalg}$, where the $a$-th and $b$-th entries on sub-channel $m$ are

$$
\mathbf{\amalg}=\left[\begin{array}{cccc}
\frac{\partial^{2} \mathcal{L}(.)}{\partial^{2} p_{1, m}} \frac{\partial^{2} \mathcal{L}(.)}{\partial p_{1, m} \partial p_{2, m}} & \cdots & \frac{\partial^{2} \mathcal{L}(.)}{\partial p_{1} \partial p_{k, m}} \\
\frac{\partial^{2} \mathcal{L}(.)}{\partial p_{2, m} \partial p_{1, m}} \frac{\partial^{2} \mathcal{L}(.)}{\partial^{2} p_{2, m}} & \cdots & \frac{\partial^{2} \mathcal{L}(.)}{\partial p_{2, m} \partial p_{k, m}} \\
\vdots & \vdots & \ddots & \vdots \\
\frac{\partial^{2} \mathcal{L}(.)}{\partial p_{k, m} \partial p_{1, m}} \frac{\partial^{2} \mathcal{L}(.)}{\partial p_{k, m} \partial p_{2, m}} & \cdots & \frac{\partial^{2} \mathcal{L}(.)}{\partial^{2} p_{k, m}}
\end{array}\right]_{\mathcal{S}_{m} \times \mathcal{S}_{m}}
$$

where $\mathcal{L}($.$) in (18) represents the Lagrangian function of (P2) and can be derived as$

$$
\begin{aligned}
& \mathcal{L}\left(p_{k}, \lambda_{k}, \eta_{k}, \pi_{k}\right)=\sum_{m=1}^{M} \sum_{k=1}^{K} p_{k, m}+\sum_{k=1}^{K} \lambda_{k}\left(\Gamma_{\min }-\sum_{m=1}^{M} R_{k, m}\right) \\
& +\sum_{k=1}^{K} \eta_{k}\left(\sum_{m=1}^{M} \sum_{l=1}^{k-1} p_{l, m}+\frac{\beta}{\left|h_{k-1, m}\right|^{2}}-p_{k, m}\right) \\
& +\sum_{k=1}^{K} \pi_{k}\left(\sum_{m=1}^{M} p_{k, m}-P_{\max }\right),
\end{aligned}
$$


where $\lambda_{k}, \eta_{k}$ and $\pi_{k}$ are the Lagrangian multipliers. The $a$-th and $b$-th entries of $\amalg$ can be computed as

$$
\mathbf{\amalg}=\left\{\begin{array}{ll}
\phi_{3}, & \text { if } a<b, \\
\phi_{4}, & \text { otherwise, }
\end{array},\right.
$$

where the values of $\phi_{3}$ and $\phi_{4}$ in (20) are given as

$\phi_{3}=$

$$
\frac{\lambda_{i}\left(\left|h_{i, m}\right|^{2} \sum_{l=1}^{i-1} p_{l, m}+\rho^{2}+p_{i, m}\left|h_{i, m}\right|^{2}\right)\left(\left|h_{i, m}\right|^{2} \sum_{l=1}^{i-1} p_{l, m}+\rho^{2}\right)\left|h_{i, m}\right|^{4}-p_{i, m}\left|h_{i, m}\right|^{4}\left(\left|h_{i, m}\right|^{2}\left(\left|h_{i, m}\right|^{2} \sum_{l=1}^{i-1} p_{l, m}+\rho^{2}\right)\right)}{\left(\left|h_{i, m}\right|^{2} \sum_{l=1}^{i-1} p_{l, m}+\rho^{2}+p_{i, m}\left|h_{i, m}\right|^{2}\left(\left|h_{i, m}\right|^{2} \sum_{l=1}^{i-1} p_{l, m}+\rho^{2}\right)^{2}\right)^{2}}
$$

$-\sum_{q=1}^{\mathcal{S}_{m}} \frac{\lambda_{q}\left(p_{q, m}\left|h_{q, m}\right|^{4}\left(2\left|h_{q, m}\right|^{4} \sum_{l=1}^{q-1} p_{l, m}+2 \rho^{2}\left|h_{q, m}\right|^{2}+p_{q, m}\left|b_{q, m}\right|^{4}\right)\right)}{\left(\left|h_{q, m}\right|^{2} \sum_{l=1}^{q-1} p_{l, m}+\rho^{2}+p_{q, m}\left|h_{q, m}\right|^{2}\left(\left|h_{q, m}\right|^{2} \sum_{l=1}^{q-1} p_{l, m}+\rho^{2}\right)^{2}\right)^{2}}$,

$$
\begin{aligned}
& \phi_{4}=\frac{\lambda_{k}\left|h_{k, m}\right|^{4}}{\left(\left|h_{k, m}\right|^{2} \sum_{l=1}^{k-1} p_{l, m}+\rho^{2}+p_{k, m}\left|h_{k, m}\right|^{2}\right)^{2}} \\
& -\sum_{j=1}^{\mathcal{S}_{m}} \frac{\lambda_{j}\left(p_{j, m}\left|h_{j, m}\right|^{4}\left(2\left|h_{j, m}\right|^{4} \sum_{l=1}^{j-1} p_{l, m}+2 \rho^{2}\left|h_{j, m}\right|^{2}+p_{j, m}\left|b_{j, m}\right|^{4}\right)\right)}{\left(\left|h_{j, m}\right|^{2} \sum_{l=1}^{j-1} p_{l, m}+\rho^{2}+p_{j, m}\left|h_{j, m}\right|^{2}\left(\left|h_{j, m}\right|^{2} \sum_{l=1}^{j-1} p_{l, m}+\rho^{2}\right)^{2}\right)^{2}}
\end{aligned}
$$

After calculating the Jacobian and Hessian matrix, now we define matrix $\Upsilon$ as

$$
\boldsymbol{\Upsilon}=\left[\begin{array}{ll}
{[\mathbf{U}]_{\mathcal{S}_{m} \times \mathcal{S}_{m}}[\mathbf{\Pi}]_{\mathcal{S}_{m} \times 3 \mathcal{S}_{m}}^{T}} \\
{[\mathbf{\Pi}]_{3 \mathcal{S}_{m} \times \mathcal{S}_{m}}[\mathbf{0}]_{3 \mathcal{S}_{m} \times 3 \mathcal{S}_{m}}}
\end{array}\right]_{4 \mathcal{S}_{m} \times 4 \mathcal{S}_{m}} .
$$

The estimates of $\left(p_{k}, \lambda_{k}, \eta_{k}, \pi_{k}\right)$ can be iteratively improved by the correction vector $\alpha=$ $\left[\left(\alpha_{p}^{z}\right)^{T}\left(\alpha_{\lambda}^{z}\right)^{T}\left(\alpha_{\eta}^{z}\right)^{T}\left(\alpha_{\pi}^{z}\right)^{T}\right]^{T}$ as

$$
\left[\begin{array}{l}
\boldsymbol{p}^{z+1} \\
\boldsymbol{\lambda}^{z+1} \\
\boldsymbol{\eta}^{z+1} \\
\boldsymbol{\pi}^{z+1}
\end{array}\right]=\left[\begin{array}{l}
\boldsymbol{p}^{z}+\psi_{p}^{z} \alpha_{p}^{z} \\
\boldsymbol{\lambda}^{z}+\psi_{\lambda}^{z} \alpha_{\lambda}^{z} \\
\boldsymbol{\eta}^{z}+\psi_{\eta}^{z} \alpha_{\eta}^{z} \\
\boldsymbol{\pi}^{z}+\psi_{\pi}^{z} \alpha_{\pi}^{z}
\end{array}\right]
$$




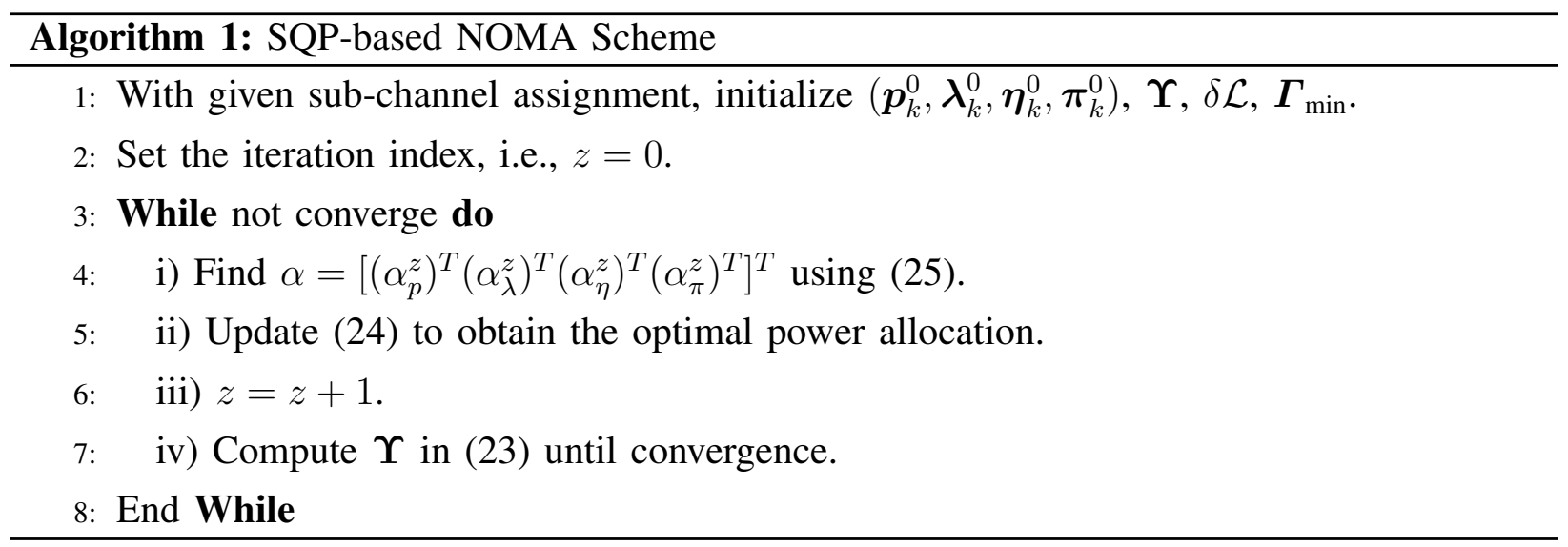

where $\psi_{p}^{z}, \psi_{\lambda}^{z}, \psi_{\eta}^{z}$ and $\psi_{\pi}^{z}$ are the non-negative step size. Finally, the vector $\alpha$ can be computed as

$$
\alpha=-\left[\delta \mathcal{L}(\boldsymbol{p})^{T} \delta \mathcal{L}(\boldsymbol{\lambda})^{T} \delta \mathcal{L}(\boldsymbol{\eta})^{T} \delta \mathcal{L}(\boldsymbol{\pi})^{T}\right]^{T} \times \boldsymbol{\Upsilon}^{-1},
$$

where $\delta \mathcal{L}(\boldsymbol{p}), \delta \mathcal{L}(\boldsymbol{\lambda}), \delta \mathcal{L}(\boldsymbol{\eta})$ and $\delta \mathcal{L}(\boldsymbol{\pi})$ are the Gradients of (19) and can be given as

$$
\begin{gathered}
\delta \mathcal{L}\left(p_{k}\right)=-\lambda_{k} \phi_{5}+\sum_{l=k+1}^{\mathcal{S}_{m}} \lambda_{l} \phi_{6}-\eta_{k}+\pi_{k}, \forall m, \\
\delta \mathcal{L}\left(\lambda_{k}\right)=\Gamma_{\min }-R_{k, m}, \forall m, \forall k, \\
\delta \mathcal{L}\left(\eta_{k}\right)=\sum_{l=1}^{k-1} p_{l, m}+\frac{\beta}{\left|h_{k-1, m}\right|^{2}}-p_{k, m}, \forall m, \\
\delta \mathcal{L}\left(\pi_{k}\right)=\sum_{m=1}^{M} \sum_{k=1}^{K} p_{k, m}-P_{\max },
\end{gathered}
$$

where $\phi_{5}$ and $\phi_{6}$ in (26) are, respectively, written as

$$
\begin{gathered}
\phi_{5}=\frac{\left|h_{k, m}\right|^{2}}{\left(\left|h_{k, m}\right|^{2} \sum_{l=1}^{k-1} p_{l, m}+\rho^{2}\right) p_{k, m}\left|h_{k, m}\right|^{2}}, \\
\phi_{6}=\frac{p_{j, m}\left|h_{j, m}\right|^{4}}{\left(\left|h_{j, m}\right|^{2} \sum_{l=1}^{j-1} p_{l, m}+\rho^{2}\right)\left(\left|h_{j, m}\right|^{j} \sum_{l=1}^{j-1} p_{l, m}+\rho^{2}+p_{j, m}\left|h_{j, m}\right|^{2}\right)} .
\end{gathered}
$$


Now we discuss the complexity of the proposed SQP-based power optimization scheme in terms of $z$ (number of iterations) required for convergence depends on the number of total available sub-channels in IoT network, i.e., $M$ and the number of IoT devices on each subchannel, i.e., $\mathcal{S}_{m}$. The number of iterations needed for convergence increases when the number of sub-channels or the number of users on each sub-channel increase. If the maximum number of IoT devices that a single sub-channel can accommodate is $\mathcal{S}_{\max }$. Then the complexity of computing optimization problem (P1) employing SQP-based power optimization scheme in iteration $z$ is $\mathcal{O}\left(M \mathcal{S}_{\max }^{2}\right)$. In what follows, assume that the total number of iterations is $Z$, the total computational complexity of the proposed scheme becomes $\mathcal{O}\left(Z M \mathcal{S}_{\max }^{2}\right)$. The detail steps of our proposed SQP-based power optimization are shown in Algorithm 1.

\section{B. KKT-based Suboptimal Power Allocation}

Now, we employ a suboptimal solution to our proposed NOMA-enabled IoT network. To do so, we apply the KKT-based conditions to (19) as

$$
\begin{aligned}
\frac{\partial \mathcal{L}(.)}{\partial p_{k, m}} & =\frac{\partial}{\partial p_{k, m}}\left[\sum_{m=1}^{M} \sum_{k=1}^{K} p_{k, m}+\sum_{k=1}^{K} \lambda_{k}\left(\Gamma_{\min }-\sum_{m=1}^{M} R_{k, m}\right)\right. \\
& +\sum_{k=1}^{K} \eta_{k}\left(\sum_{m=1}^{M} \sum_{l=1}^{k-1} p_{l, m}+\frac{\beta}{\left|h_{k-1, m}\right|^{2}}-p_{k, m}\right) \\
& \left.+\sum_{k=1}^{K} \pi_{k}\left(\sum_{m=1}^{M} p_{k, m}-P_{\max }\right)\right]
\end{aligned}
$$

After taking derivations and some modifications, we get

$$
\frac{\partial \mathcal{L}(.)}{\partial p_{k, m}}=\frac{\lambda_{k} \varrho_{k, m}}{\ln _{2}\left(1+p_{k, m} \varrho_{k, m}\right)}-\delta_{k, m}-\eta_{k}+\pi_{k},
$$

where $\varrho_{k, m}$ and $\delta_{k, m}$ can be defined as

$$
\begin{gathered}
\varrho_{k, m}=\frac{\left|h_{k, m}\right|^{2}}{\left|h_{k, m}\right|^{2} \sum_{l=1}^{k-1} p_{l, m}+\rho^{2}}, \\
\delta_{k, m}=\sum_{l=1}^{k-1} \frac{\xi_{l, m}\left|h_{l, m}\right|^{2}}{\ln _{2}\left(1+\xi_{l, m}\right) \sum_{i=1}^{l-1} p_{i, m}\left|h_{l, m}\right|^{2}} .
\end{gathered}
$$


By setting $\frac{\partial \mathcal{L}(.)}{\partial p_{k, m}}=0$, the transmit power of IoT device $k$ on sub-channel $m$ can be obtained as

$$
p_{k, m}^{*}=\left[\frac{\lambda_{k, m}}{\delta_{k, m}+\eta_{k}-\pi_{k}}-\frac{1}{\mathcal{O}_{k, m}}\right]^{+}
$$

where $(\Lambda)^{+}=\max (0, \Lambda)$. We iteratively update $\lambda_{k}, \eta_{k}$ and $\pi_{k}$ using sub-gradient method as [42], [43]

$$
\begin{gathered}
\lambda_{k}(z+1)=\left[\lambda_{k}(z)+\psi(z)\left(\Gamma_{\min }-R_{k, m}\right)\right]^{+}, \forall k, \forall m, \\
\eta_{k}(z+1)=\left[\eta_{k}(z)+\psi(z)\left(\sum_{l=1}^{k-1} p_{l, m}+\frac{\beta}{\left|h_{k-l, m}\right|^{2}}-p_{k, m}\right)\right]^{+}, \forall m, \\
\pi_{k}(z+1)=\left[\pi_{k}(z)+\psi(z)\left(\sum_{m=1}^{M} \sum_{k=1}^{K} p_{k, m}-P_{\max }\right)\right]^{+},
\end{gathered}
$$

In each $z$, we first update $\lambda_{k}, \eta_{k}$ and $\pi_{k}$ by $p_{k, m}^{*}$, and then use optimal values of $\lambda_{k}, \eta_{k}$ and $\pi_{k}$ in the subsequent iterations to obtain $p_{k, m}^{*}$. The analysis of complexity of KKT-based approach can be found in $[10]^{2}$ and Algorithm 2 provides the complete steps of the benchmark KKT-based power allocation scheme.

\section{RESUlTS AND Discussion}

This section provides the simulations results and their discussion. Unless mentioned otherwise, the details of the simulation parameters are as follows: the maximum number of IoT devices and sub-channels are set as $K=24$ and $M=12$, respectively. The maximum power budget at $\mathrm{BS}$ is set as $P_{\max }=20 \mathrm{~W}$ while the minimum QoS threshold is set as $\tau_{\min }=1 \mathrm{~b} / \mathrm{s} / \mathrm{Hz}$. The maximum number of IoT devices on each sub-channel are $\mathcal{S}_{\max }=2$ and the circuit power is $\mathrm{Pc}=0.5 \mathrm{~W}$. Moreover, the minimum power gap among different IoT devices for successful SIC on each sub-channel is set as $\beta=0.2 \mathrm{~W}$ while the variance of additive white Gaussian noise is $\rho^{2}=0.1$. Also, we consider Rayleigh fading and obtain the average results from $10^{4}$ Monte Carlo realizations.

\footnotetext{
${ }^{2}$ Although the SQP-based approach is somewhat more complex in comparison to the benchmark KKT-based approach, the overall performance gain (as shown in Section-IV) is considerably high. Due to this reason, it can satisfactorily be used to improve the performance of NOMA-enabled IoT networks
} 

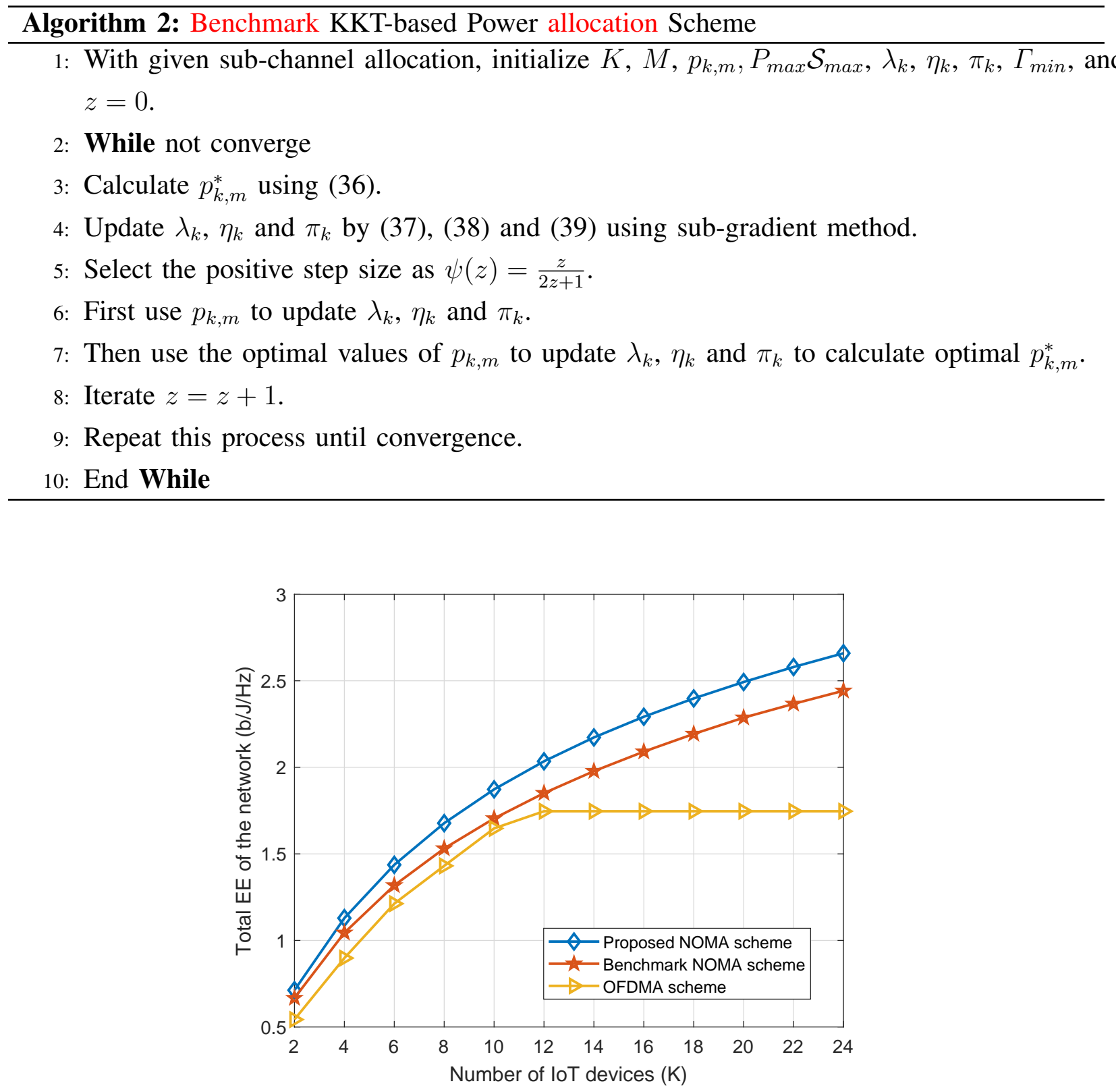

Figure 2. Total Achievable System EE versus varying number of IoT devices.

In this work, the achievable EE $(\mathrm{b} / \mathrm{J} / \mathrm{Hz})$ of the proposed IoT network is computed as the sum rate of IoT devices $(\mathrm{b} / \mathrm{s} / \mathrm{Hz})$ divided by the total power consumption $(\mathrm{W})$ of the network. It can 


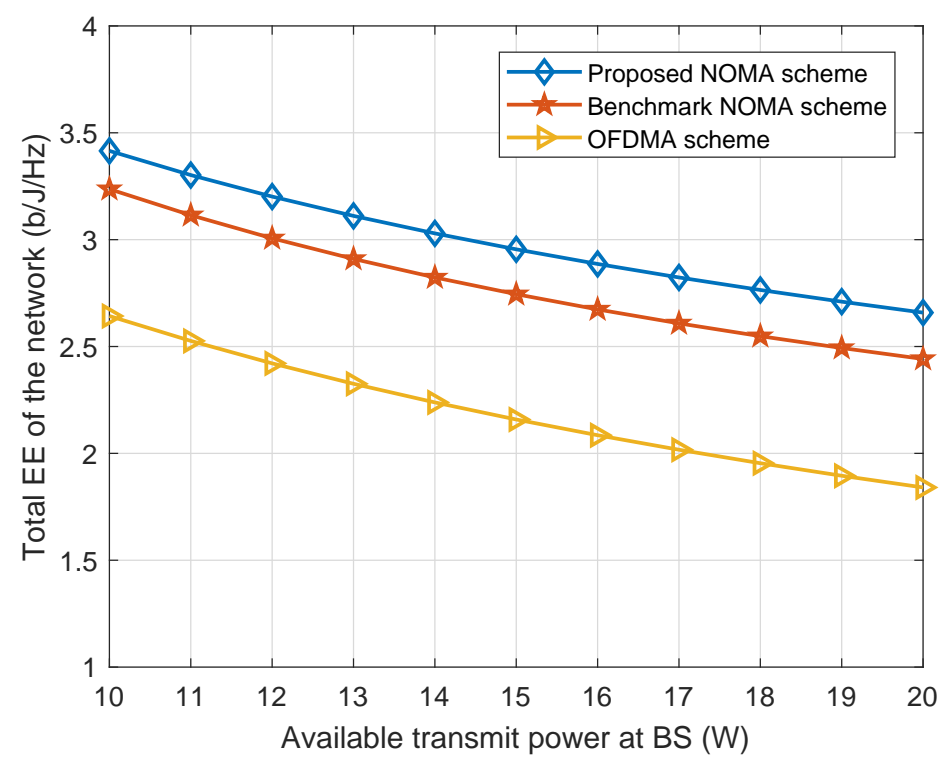

Figure 3. Total Achievable System EE versus varying BS transmission power.

be mathematically formulated as

$$
\mathrm{EE}=\frac{\sum_{m=1}^{M} \sum_{k=1}^{K} \log _{2}\left(1+\xi_{k, m}\right)}{\sum_{m=1}^{M} \sum_{k=1}^{K} p_{k, m}+\mathrm{Pc}}(\mathrm{b} / \mathrm{J} / \mathrm{Hz}) .
$$

Our simulation results compare the following three schemes:

1) Proposed NOMA scheme: This is the proposed optimal SQP-based power allocation scheme as discussed in Section III-A.

2) Benchmark NOMA scheme: It denotes the benchmark suboptimal KKT-based power allocation scheme as provided in Section III-B.

3) OFDMA scheme: This represents the traditional OMA power allocation scheme, where a sub-channel can accommodate only one user at one time.

Fig. 2 depicts the achievable system EE versus the increasing number of IoT devices. We can observe that the proposed SQP-based optimization scheme outperforms the suboptimal benchmark NOMA scheme and the conventional OFDMA scheme. For the smaller numbers of IoT devices, the difference between the achievable EE of the proposed SQP-based and benchmark KKT-based schemes are negligible. However, as the number of IoT devices increase, 


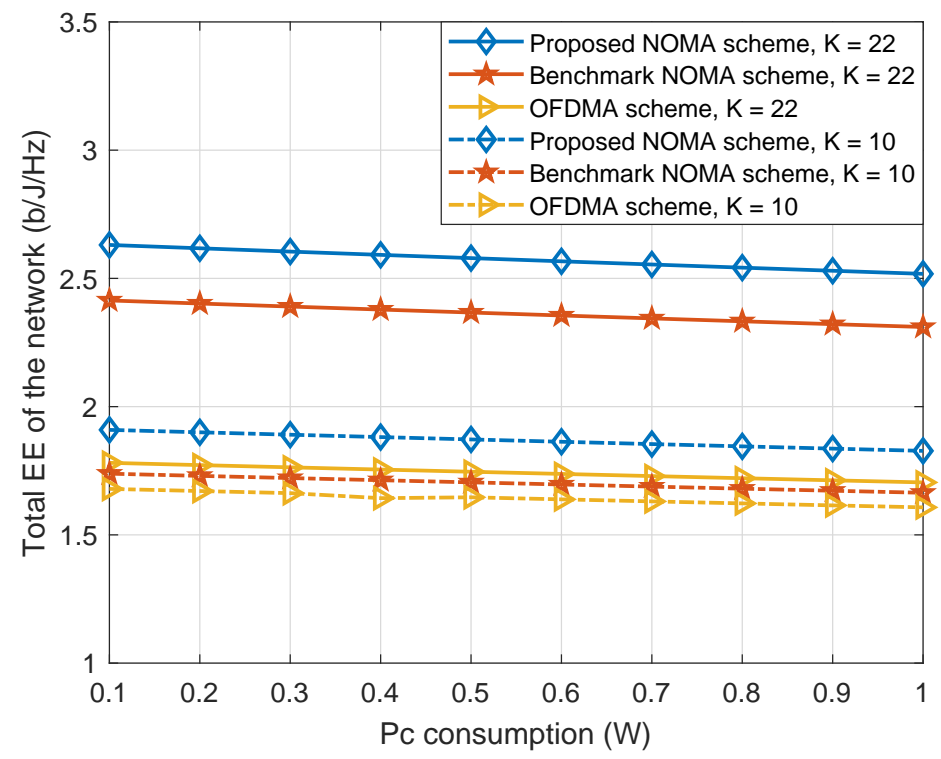

Figure 4. Impact of the circuit power $\left(P_{C}\right)$ consumption on the total achievable EE of the IoT network.

the difference between the curves of the proposed SQP-based NOMA scheme and benchmark KKT-based NOMA scheme increases significantly such that it grows to almost $0.5 \mathrm{bit} / \mathrm{J} / \mathrm{Hz}$ when the total number of IoT devices approaches 24. On the other end, the OFDMA based scheme performs very poorly for large number of IoT devices. It is evident from the figure that the achievable EE of the OFDMA scheme saturates as the number of IoT devices reaches to 12. It is because OFDMA based scheme can accommodate exactly the same number of IoT devices as the number of sub-channels, which is considered 12 in our proposed framework. This illustrates the inability of OFDMA scheme to handle a large number of IoT devices, while both NOMA schemes (proposed SQP-based and benchmark KKT-based) continues to improve as the number of IoT devices increases beyond 12 .

To further investigate the performance of our proposed SQP-based scheme, it is important to observe the impact of the varying available transmission budget at BS on the achievable EE. Fig. 3 describes the achievable EE versus the available power budget at the BS. It demonstrates that the curves for both NOMA and OFDMA based schemes decreases with an increase in the available power budget at the BS. From Eq. (40), we can know that the achievable EE follows a bell shaped curve in which it increases with an increase in the transmission power budget 


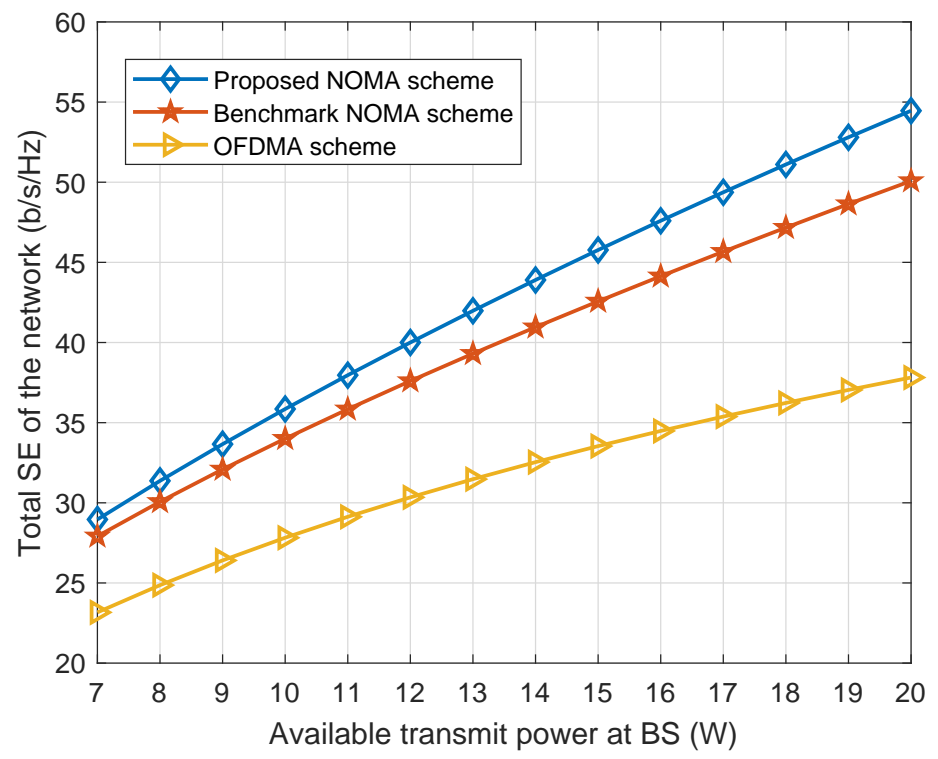

Figure 5. Effect of available transmit power at BS on the total achievable SE of IoT network.

until a saturating point and afterwards it starts decreasing with an increase in the transmitted power. However, we can observe that the gap between the curves for the proposed NOMA and OFDMA based schemes are considerably large, which indicates the poor performance of the OFDMA based scheme. In addition, we note that the proposed SQP-based NOMA approach shows significantly improved performance as compared to the benchmark KKT-based NOMA and OFDMA schemes, for the increasing BS available transmission power budget.

Fig. 4 illustrates an achievable EE as a function of the BS circuit power $\left(P_{C}\right)$ consumption. It can be observed that an increase in $P_{C}$ consumption linearly decreases the achievable EE. Apparently, the OFDMA based scheme consumes significantly large transmission power for efficient operation rapidly decreasing the achievable EE in contrast to the benchmark suboptimal KKT-based NOMA scheme and proposed SQP-based NOMA scheme. It can also been seen that with an increase in the number of IoT devices increases the achievable system EE. However, for a low number of IoT devices, the curves of the benchmark NOMA and OFDMA schemes are close to each other. In every case, the proposed SQP-based NOMA scheme outperforms the other two schemes, showing that the proposed SQP-based NOMA scheme achieves overall better achievable EE for a larger number of energy-constrained IoT devices. 
Fig. 5 investigates the achievable system SE versus varying the transmit power at the BS. We can also observe that an increase in the available transmission budget at BS, the achievable system SE increases. More specifically, the difference in the achievable system SE curves between the proposed SQP-based NOMA scheme and OFDMA based scheme increases at the higher values of available transmission power of BS. The same intuition can also be observed by comparing the achievable system SE of the proposed SQP-based NOMA scheme versus the benchmark KKT-based NOMA scheme. However, in any case, the proposed SQP-based power allocation scheme provides considerable improvements in achievable system SE over OFDMA based and the benchmark KKT-based NOMA schemes.

Fig. 6 analyses the achievable system SE versus the increasing number of IoT devices for the proposed SQP-based NOMA scheme in contrast to the benchmark KKT-based NOMA scheme and OFDMA based scheme. It is evident from the figure, the overall achievable SE increases with an increase in the number of IoT devices, since more users can be accommodated under the same network resources. The proposed SQP-based NOMA scheme outperforms the benchmark KKT-based NOMA power allocation approach. In contrast, the performance of the OFDMA scheme initially grows and becomes saturated as the number of IoT devices reaches to 12 . This is due to the fact that the available spectrum resource are limited to accommodate high number of IoT devices using OFDMA. This shows that the proposed SQP-based NOMA scheme is better at accommodating a larger number of IoT devices.

\section{CONCLUSION}

NOMA is considered as a key enabler to manage the massive number of IoT devices in the future generation systems. This work has proposed an energy-efficient scheme for power allocation in NOMA-enabled IoT networks. Firstly, we have formulated a non-convex optimization problem of power allocation under the SIC and QoS constraints. Secondly, we have adopted the proposed SQP-based scheme to obtain a reliable solution for the non-convex power allocation problem in NOMA-enabled IoT networks. Finally, we have compared the the performance evaluation of the proposed SQP-based power allocation scheme with the conventional KKT-based NOMA and OFDMA based schemes. As evident by the performance evaluation, our proposed SQP-based scheme outperforms benchmark KKT-based NOMA scheme. We would like to do further future work to investigate the impact of mobility among IoT devices on the achievable EE and SE for 


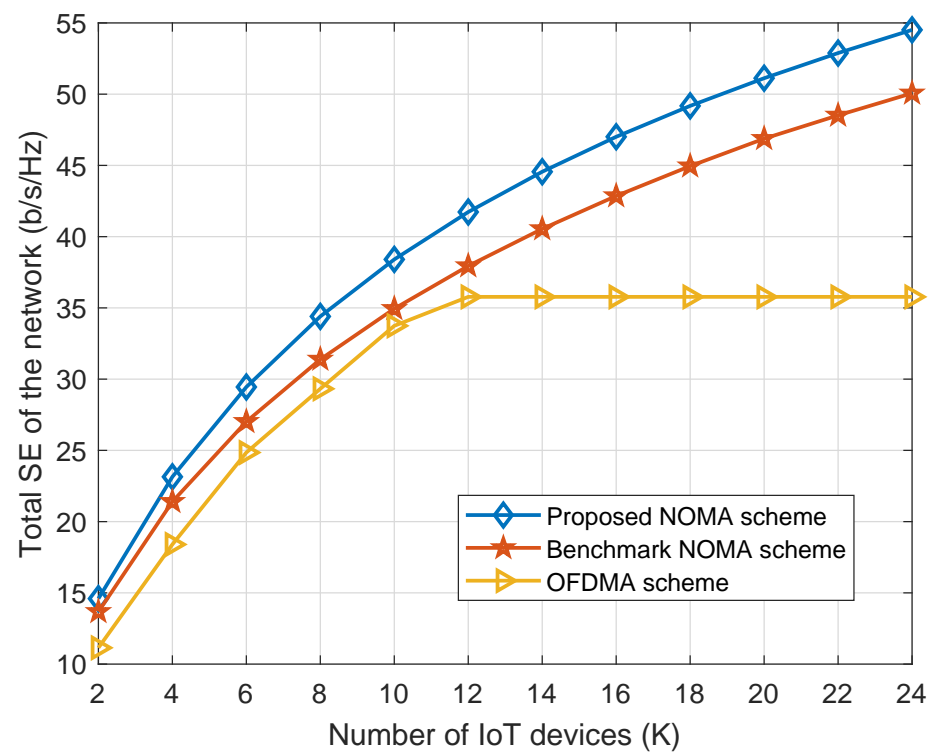

Figure 6. Total Achievable SE as a function of number of varying IoT devices.

the proposed SQP-based NOMA scheme in contrast to the benchmark KKT-based and OFDMA based schemes.

\section{REFERENCES}

[1] Q. Wu, W. Chen, D. W. K. Ng, and R. Schober, "Spectral and energy-efficient wireless powered IoT networks: NOMA or TDMA?” IEEE Trans. Vehic. Techn., vol. 67, no. 7, pp. 6663-6667, 2018.

[2] M. Shirvanimoghaddam, M. Dohler, and S. J. Johnson, "Massive non-orthogonal multiple access for cellular IoT: Potentials and limitations," IEEE Commun. Mag., vol. 55, no. 9, pp. 55-61, 2017.

[3] S. Qureshi, S. A. Hassan, and D. N. K. Jayakody, "Divide-and-allocate: An uplink successive bandwidth division NOMA system,” Trans. Emerg. Telecommun. Technol., vol. 29, no. 1, p. e3216, 2018.

[4] T. Jabeen, Z. Ali, W. U. Khan, F. Jameel, I. Khan, G. A. S. Sidhu, and B. J. Choi, "Joint Power Allocation and Link Selection for Multi-Carrier Buffer Aided Relay Network,” Electronics, vol. 8, no. 6, p. 686, 2019.

[5] F. C. Ribeiro, J. Guerreiro, R. Dinis, F. Cercas, and D. N. K. Jayakody, "Multi-user detection for the downlink of NOMA systems with multi-antenna schemes and power-efficient amplifiers," Physical Commun., vol. 33, pp. 199-205, 2019.

[6] Z. Ali, G. A. S. Sidhu, M. Waqas, and F. Gao, "On fair power optimization in nonorthogonal multiple access multiuser networks," Trans. Emerg. Telecommun. Techn., vol. 29, no. 12, p. e3540, 2018.

[7] W. U. Khan, F. Jameel, T. Ristaniemi, S. Khan, G. A. S. Sidhu, and J. Liu, "Joint spectral and energy efficiency optimization for downlink NOMA networks," IEEE Trans. Cogn. Commun. Netw., pp. 1-1, 2019.

[8] F. Jameel, S. Wyne, G. Kaddoum, and T. Q. Duong, “A comprehensive survey on cooperative relaying and jamming strategies for physical layer security," IEEE Commun. Surv. Tut., pp. 1-1, 2018. 
[9] Z. Ali, Y. Rao, W. U. Khan, and G. A. S. Sidhu, "Joint user pairing, channel assignment and power allocation in NOMA based CR systems," Appl. Sciences, vol. 9, no. 20, p. 4282, 2019.

[10] W. U. Khan, Z. Yu, S. Yu, G. A. S. Sidhu, and J. Liu, "Efficient power allocation in downlink multi-cell multi-user NOMA networks," IET Commun., vol. 13, no. 4, pp. 396-402, 2019.

[11] A. E. Mostafa, Y. Zhou, and V. W. Wong, "Connectivity maximization for narrowband IoT systems with NOMA," in Proc. IEEE Int. Conf. Commun. (ICC). IEEE, 2017, pp. 1-6.

[12] X. Li, M. Zhao, C. Zhang, W. U. Khan, J. Wu, K. M. Rabie, and R. Kharel, "Security Analysis of Multi-Antenna NOMA Networks Under I/Q Imbalance,” Electronics, vol. 8, no. 11, p. 1327, 2019.

[13] A. Ali, A. Baig, G. M. Awan, W. U. Khan, Z. Ali, and G. A. S. Sidhu, "Efficient resource management for sum capacity maximization in 5G NOMA systems," Appl. Syst. Innov., vol. 2, no. 3, p. 27, 2019.

[14] B. Su, Q. Ni, W. Yu, and H. Pervaiz, "Outage constrained robust beamforming design for SWIPT-enabled cooperative NOMA system,” in Proc. IEEE Int. Conf. Commun. (ICC), May 2019, pp. 1-6.

[15] F. Jameel, S. Wyne, S. J. Nawaz, Z. Chang, and T. Ristaniemi, "Outage analysis of relay-aided non-orthogonal multiple access with partial relay selection," in Proc. IEEE Glob. Work. (GC Wkshps), Dec 2018, pp. 1-6.

[16] W. Shin, M. Vaezi, B. Lee, D. J. Love, J. Lee, and H. V. Poor, "Non-orthogonal multiple access in multi-cell networks: Theory, performance, and practical challenges," IEEE Commun. Mag., vol. 55, no. 10, pp. 176-183, 2017.

[17] J. Choi, "Non-orthogonal multiple access in downlink coordinated two-point systems," IEEE Commun. Lett., vol. 18, no. 2, pp. 313-316, 2014.

[18] Y. Tian, A. R. Nix, and M. Beach, "On the performance of opportunistic NOMA in downlink CoMP networks," IEEE Commun. Lett., vol. 20, no. 5, pp. 998-1001, 2016.

[19] A. Beylerian and T. Ohtsuki, "Coordinated non-orthogonal multiple access (CO-NOMA)," in 2016 IEEE Global Communications Conference (GLOBECOM). IEEE, 2016, pp. 1-5.

[20] Y. Tian, A. Nix, and M. Beach, "On the performance of a multi-tier NOMA strategy in coordinated multi-point networks," IEEE Commun. Lett., vol. 21, no. 11, pp. 2448-2451, 2017.

[21] Z. Yang, C. Pan, W. Xu, Y. Pan, M. Chen, and M. Elkashlan, "Power control for multi-cell networks with non-orthogonal multiple access," IEEE Trans. Wireless Commun., vol. 17, no. 2, pp. 927-942, 2017.

[22] S. H. Amin, A. H. Mehana, S. S. Soliman, and Y. A. Fahmy, "Power allocation for maximum MIMO-NOMA system user-rate," in Proc. IEEE Glob. Workshops (GC Wkshps). IEEE, 2018, pp. 1-6.

[23] X. Sun, N. Yang, S. Yan, Z. Ding, D. W. K. Ng, C. Shen, and Z. Zhong, "Joint beamforming and power allocation in downlink NOMA multiuser MIMO networks,” IEEE Trans. Wireless Commun., vol. 17, no. 8, pp. 5367-5381, 2018.

[24] M. Zeng, A. Yadav, O. A. Dobre, and H. V. Poor, "Energy-efficient power allocation for MIMO-NOMA with multiple users in a cluster," IEEE Access, vol. 6, pp. 5170-5181, 2018.

[25] S. R. Islam, M. Zeng, O. A. Dobre, and K.-S. Kwak, "Resource allocation for downlink NOMA systems: Key techniques and open issues," IEEE Wireless Commun., vol. 25, no. 2, pp. 40-47, 2018.

[26] J. Shi, W. Yu, Q. Ni, W. Liang, Z. Li, and P. Xiao, "Energy efficient resource allocation in hybrid non-orthogonal multiple access systems," IEEE Trans. Commun., vol. 67, no. 5, pp. 3496-3511, May 2019.

[27] X. Song, L. Dong, J. Wang, L. Qin, and X. Han, "Energy efficient power allocation for downlink NOMA heterogeneous networks with imperfect CSI," IEEE Access, vol. 7, pp. 39329-39340, 2019.

[28] F. Fang, J. Cheng, and Z. Ding, "Joint energy efficient subchannel and power optimization for a downlink NOMA heterogeneous network," IEEE Trans. Vehic. Techn., vol. 68, no. 2, pp. 1351-1364, Feb 2019. 
[29] D. Zhai, R. Zhang, L. Cai, B. Li, and Y. Jiang, "Energy-Efficient User Scheduling and Power Allocation for NOMA-Based Wireless Networks With Massive IoT Devices,” IEEE Internet of Things J., vol. 5, no. 3, pp. 1857-1868, June 2018.

[30] M. Basharat, W. Ejaz, M. Naeem, A. M. Khattak, A. Anpalagan, and O. Alfandi, "Energy efficient resource allocation for NOMA in cellular IoT with energy harvesting," in Proc. 13th IEEE Int. Conf. on Emerging Technologies (ICET), Dec 2017, pp. 1-6.

[31] D. Sikeridis, E. E. Tsiropoulou, M. Devetsikiotis, and S. Papavassiliou, "Energy-Efficient Orchestration in Wireless Powered Internet of Things Infrastructures," IEEE Trans. on Green Commun. Netw., vol. 3, no. 2, pp. 317-328, June 2019.

[32] Z. Yang, W. Xu, Y. Pan, C. Pan, and M. Chen, "Energy Efficient Resource Allocation in Machine-to-Machine Communications With Multiple Access and Energy Harvesting for IoT," IEEE Internet of Things J., vol. 5, no. 1, pp. 229-245, Feb 2018.

[33] Y. Wu, B. Shi, L. P. Qian, F. Hou, J. Cai, and X. Shen, "Energy-Efficient Multi-Task Multi-access Computation Offloading via NOMA Transmission for IoTs,” IEEE Trans. on Indust. Infor., pp. 1-1, 2019.

[34] S. Han, X. Xu, S. Fang, Y. Sun, Y. Cao, X. Tao, and P. Zhang, "Energy Efficient Secure Computation Offloading in NOMA-Based mMTC Networks for IoT," IEEE Internet of Things J., vol. 6, no. 3, pp. 5674-5690, June 2019.

[35] Z. Li and J. Gui, "Energy-Efficient Resource Allocation With Hybrid TDMA-NOMA for Cellular-Enabled Machine-toMachine Communications,” IEEE Access, vol. 7, pp. 105 800-105 815, 2019.

[36] B. Di, L. Song, and Y. Li, "Sub-channel assignment, power allocation, and user scheduling for non-orthogonal multiple access networks," IEEE Trans. Wireless Commun., vol. 15, no. 11, pp. 7686-7698, 2016.

[37] S. Boyd and L. Vandenberghe, Convex optimization. Cambridge university press, 2004.

[38] J. Zhao, Y. Liu, K. K. Chai, A. Nallanathan, Y. Chen, and Z. Han, "Spectrum allocation and power control for nonorthogonal multiple access in HetNets," IEEE Trans. Wireless Commun., vol. 16, no. 9, pp. 5825-5837, 2017.

[39] W. U. Khan, Z. Ali, M. Waqas, and G. A. S. Sidhu, "Efficient power allocation with individual QoS guarantees in future small-cell networks,” AEU-Int. J. of Electr. Commun., vol. 105, pp. 36-41, 2019.

[40] P. T. Boggs and J. W. Tolle, "Sequential quadratic programming for large-scale nonlinear optimization," J. computa. appl. math., vol. 124, no. 1-2, pp. 123-137, 2000.

[41] A. Attar, M. R. Nakhai, and A. H. Aghvami, "Cognitive radio game for secondary spectrum access problem," IEEE Trans. Wireless Commun., vol. 8, no. 4, pp. 2121-2131, 2009.

[42] W. U. Khan, F. Jameel, T. Ristaniemi, B. M. Elhalawany, and J. Liu, "Efficient power allocation for multi-cell uplink NOMA network," in Proc. 89th IEEE Vehic. Techn. Conf. (VTC Spring). IEEE, 2019, pp. 1-5.

[43] W. U. Khan, "Maximizing physical layer security in relay-assisted multicarrier nonorthogonal multiple access transmission," Internet Techn. Lett., vol. 2, no. 2, p. e76, 2019. 